La Revue

des Droits

de l'Homme

\section{La Revue des droits de l'homme}

Revue du Centre de recherches et d'études sur les droits fondamentaux

Actualités Droits-Libertés | 2020

\title{
La protection de l'environnement, objectif de valeur constitutionnelle : vers une invocabilité asymétrique de certaines normes constitutionnelles?
}

Remarques sur la décision $n^{\circ}$ 2019-823 QPC du 31 janvier 2020, Union des industries de la protection des plantes

Véronique Champeil-Desplats

\section{(2) OpenEdition}

Journals

Electronic version

URL: http://journals.openedition.org/revdh/8629

DOI: $10.4000 /$ revdh.8629

ISSN: 2264-119X

Publisher

Centre de recherches et d'études sur les droits fondamentaux

Electronic reference

Véronique Champeil-Desplats, «La protection de l'environnement, objectif de valeur constitutionnelle vers une invocabilité asymétrique de certaines normes constitutionnelles ? », La Revue des droits de I'homme [Online], Actualités Droits-Libertés, Online since 24 February 2020, connection on 06 November 2020. URL : http://journals.openedition.org/revdh/8629; DOI : https://doi.org/10.4000/ revdh.8629

This text was automatically generated on 6 November 2020 .

Tous droits réservés 


\section{La protection de l'environnement, objectif de valeur constitutionnelle : vers une invocabilité asymétrique de certaines normes constitutionnelles?}

Remarques sur la décision n²019-823 QPC du 31 janvier 2020, Union des industries de la protection des plantes

\section{Véronique Champeil-Desplats}

1 Nul doute que la décision $n^{\circ} 2019-823$ QPC du 31 janvier 2020 fera date. Alors que le Conseil constitutionnel avait estimé quelques années plus tôt qu' «aucun» des sept alinéas du préambule de la Charte de l'environnement - auxquels il reconnait pourtant par ailleurs une valeur constitutionnelle (décision n²008-564 DC, 19 juin 2008) - « n'institue un droit ou une liberté que la Constitution garantit " pouvant être invoqué « à l'appui d'une question prioritaire de constitutionnalité (QPC)» (décision n² 2014-394 QPC du 7 mai 2014, Société Casuca) ${ }^{1}$, il admet dans la décision commentée qu'il découle de ce même préambule que «la protection de l'environnement, patrimoine commun des êtres humains, constitue un objectif de valeur constitutionnelle » (I).

2 Voilà qui pourra réjouir toutes celles et tous ceux qui ont toujours refusé de réduire la Charte de l'environnement, y compris son préambule, à un texte purement symbolique ou programmatique ${ }^{2}$. Cette consécration de l'utilité juridique de l'incipit augure-t-elle pour autant un changement de conception quant à son invocabilité par des requérants dans le cadre de la QPC ? Autrement dit, l'invocation du préambule de la charte de l'environnement par le Conseil constitutionnel pour justifier son appréciation de la constitutionnalité de dispositions législatives contestées à l'occasion d'une QPC emporte-t-elle la possibilité de son invocabilité par des justiciables ? Rien n'est certain (II). 


\section{I/ - La protection de l'environnement : d'un objectif d'intérêt général à un objectif de valeur constitutionnelle.}

3 Le Conseil constitutionnel ne se contente pas dans sa décision du 31 janvier 2020 d'affirmer solennellement qu'il découle des termes du préambule de la charte de l'environnement que « la protection de l'environnement, patrimoine commun des êtres humains, constitue un objectif de valeur constitutionnelle ». Il rappelle également, sous une forme analogue, qu'il découle du onzième alinéa du préambule de la Constitution de 1946 un objectif de valeur constitutionnelle de protection de la santé. Il conclut de la lecture conjointe de ces deux objectifs qu'il appartient au législateur d'assurer leur conciliation «avec l'exercice de la liberté d'entreprendre» qu'invoquait, en l'occurrence, l'Union des industries de la protection des plantes à l'appui de sa QPC contre le paragraphe IV de l'article L. 253-8 du code rural et de la pêche maritime. Celui-ci interdit, «à compter du 1er janvier 2022 la production, le stockage et la circulation de produits phytopharmaceutiques contenant des substances actives non approuvées pour des raisons liées à la protection de la santé humaine ou animale ou de l'environnement conformément au règlement (CE) $n^{\circ}$ 1107/2009 du Parlement européen et du Conseil du 21 octobre 2009 précitée, sous réserve du respect des règles de l'Organisation mondiale du commerce ».

Bien plus, le Conseil constitutionnel précise que le législateur "est fondé à tenir compte des effets que les activités exercées en France peuvent porter à l'environnement à l'étranger ». L'énoncé du nouvel objectif à valeur constitutionnelle de protection du l'environnement, la précision de cette large portée, sa combinaison avec l'objectif de protection de la santé et le rappel de l'exigence de conciliation de normes de valeur constitutionnelle justifiant des visées contradictoires permettent alors au Conseil de rejeter le recours de la partie requérante. Il conclut que l'interdiction «d'exportation de certains produits phytopharmaceutiques contenant des substances actives non approuvées par l'Union européenne » n'est pas contraire à la constitution.

5 La consécration de la protection de l'environnement en tant qu'objectif à valeur constitutionnelle fait suite à sa qualification - dont on ignorait encore le caractère annonciateur - quelques semaines plus tôt d'objectif d'intérêt général. La référence à un tel objectif avait permis au Conseil de justifier l'exclusion « des carburants produits à partir d'huile de palme du régime favorable prévu dans le cadre de la taxe incitative relative à l'incorporation de biocarburants" (article 266 quindecies du code des douanes) (décision n 2019-808 QPC du 11 octobre 2019). Il rejetait alors le recours de la Société Total raffinage France, estimant qu'il ne lui appartenait pas «de remettre en cause l'appréciation par le législateur des conséquences pour l'environnement de la culture des matières premières en question, dès lors que cette appréciation n'est pas, en l'état des connaissances, manifestement inadéquate au regard de l'objectif d'intérêt général de protection de l'environnement poursuivi ». Le Conseil précisait même qu'« en excluant pour le calcul de la taxe la possibilité de démontrer que l'huile de palme pourrait être produite dans des conditions particulières permettant d'éviter le risque de hausse indirecte des émissions de gaz à effet de serre, le législateur a, en l'état des connaissances et des conditions mondiales d'exploitation de l'huile de palme, retenu des critères objectifs et rationnels en fonction du but poursuivi ». Le principe d'égalité 
devant les charges publiques invoqué par la société requérante n'est dès lors pas méconnu.

6 Avec la décision n ${ }^{\circ}$ 2019-823 QPC du 31 janvier 2020, la protection de l'environnement passe donc du statut d'objectif intérêt général à celui d'objectif de valeur constitutionnelle. Elle se voit aussi doter a posteriori du fondement textuel qui lui faisait défaut dans la décision du 11 octobre 2019, à savoir le préambule de la Charte de l'environnement. Il y a là un procédé de justification classique de la part du Conseil constitutionnel lorsqu'il énonce de nouvelles normes de valeur constitutionnelle. À cet égard, connaissant les précautions accrues prises par le Conseil constitutionnel depuis les années 1990 pour prendre appui sur des dispositions du texte de la constitution lorsqu'il énonce de nouveaux principes, droits et libertés, exigences ou objectifs de valeur constitutionnelle (et quel que soit le regard critique que l'on puisse porter sur la filiation établie entre le texte justificatif et l'énoncé supposé en découler), c'est l'initiative d'avoir qualifié la protection de l'environnement d'intérêt général sans assise textuelle qui avait pu paraitre surprenante.

\section{II/ - Invocation par le Conseil, invocabilité pour le justiciable?}

7 Faut-il voir dans la décision du 31 janvier 2020 une évolution quant à la portée juridique du préambule de la Charte de l'environnement? Pas nécessairement.

8 Ce n'est en effet pas la première fois que le Conseil se réfère à ce préambule dans le cadre de QPC pour en tirer des conséquences juridiques. La décision n² 2011-192 QPC du 10 novembre 2011 avait déjà admis que "le secret de la défense nationale participe de la sauvegarde des intérêts fondamentaux de la Nation, réaffirmés par la Charte de l'environnement, au nombre desquels figurent l'indépendance de la Nation et l'intégrité du territoire ». La formule fait écho au $6^{\text {ème }}$ alinéa du préambule de la Charte selon lequel «la préservation de l'environnement doit être recherchée au même titre que les autres intérêts fondamentaux de la Nation ». Toutefois, en l'espèce, ce $6^{\text {ème }}$ alinéa n'était pas invoqué «à l'appui» de la QPC soulevée, mais par le Conseil constitutionnel lui-même pour délimiter la portée des droits et libertés constitutionnellement garantis invoqués par les requérants.

Bis repetita dans la décision du 31 janvier 2020. Le Conseil confirme la possibilité qu'il se donne d'invoquer le préambule de la Charte pour, en l'espèce, justifier la formulation d'un nouvel objectif de valeur constitutionnelle ayant pour fonction de limiter la portée des droits et libertés que la constitution garantit invoqués par les justiciables. Mais cela ne signifie pas pour autant qu'il reconnaisse à ces derniers (ou plutôt à d'autres) la faculté de se prévaloir de ce préambule et des principes ou objectifs réputés en découler. Pour le moment, rien n'indique l'abandon de la solution retenue dans la QPC $\mathrm{n}^{\circ}$ 2014-394 QPC du 7 mai 2014, Société Casuca selon laquelle les alinéas du préambule de la Charte "ne peuvent être invoqués à l'appui d'une question prioritaire de constitutionnalité sur le fondement de l'article 61-1 de la Constitution».

10 Si l'on en restait là, s'accentuerait, dans le cadre des QPC, une situation d'invocabilité asymétrique de certaines normes constitutionnelles, à savoir celles que le Conseil qualifie le plus souvent d'objectifs et qu'il ne considère pas intégrer la notion de droits et libertés que la constitution garantit. Alors, en effet, que le Conseil constitutionnel 
s'accorde la possibilité d'opposer lesdites normes aux droits et libertés que les justiciables invoquent pour contester la constitutionnalité de dispositions législatives, et justifie ainsi, contra, leur validité, il refuse que celles-ci puissent être invoquées par les justiciables. En l'occurrence, plus concrètement, le Conseil constitutionnel oppose l'objectif de valeur constitutionnelle de protection de l'environnement à la partie requérante qui se fonde sur la liberté d'entreprendre pour contester la constitutionnalité de dispositions législatives, mais rien n'indique qu'une association protectrice de l'environnement, par exemple, pourrait invoquer à l'appui d'une QPC cet objectif pour contester des dispositions législatives adoptées sur le fondement de cette même liberté d'entreprendre.

11 Cette situation peut être rapprochée du sort réservé à l'objectif d'égal accès des femmes et des hommes aux responsabilités professionnelles énoncé à l'article premier alinéa 2 de la Constitution. Le Conseil constitutionnel ne l'estime pas constituer un droit ou une liberté que la Constitution garantit dont la méconnaissance peut être invoquée à l'appui QPC. Pourtant, en rappelant que cet objectif confère au législateur la faculté " d'adopter des dispositions revêtant soit un caractère incitatif, soit un caractère contraignant », le Conseil se laisse ouverte la possibilité de s'en prévaloir à l'encontre de requérants qui contestent des dispositions législatives paritaires, et ce même s'il entend le concilier avec d'autres règles et principes de valeur constitutionnelle (décision n² 2015-465 du 24 avril 2015).

Pour en revenir aux décisions du 31 janvier 2020 et du 11 octobre 2019, ceux et celles qui appelaient des signes de rééquilibrage entre les libertés économiques, d'un côté, et des exigences à caractère social ou environnemental de l'autre, ne peuvent qu'être satisfaits de la consécration constitutionnelle d'un objectif de protection de l'environnement. D'abord en tant qu'intérêt général, puis en qualité d'objectif à valeur constitutionnelle, la protection de l'environnement est opposée par le Conseil à des acteurs économiques qui se prévalaient de l'égalité devant les charges publiques et la liberté d'entreprendre.

Les usages de ce nouvel objectif restent toutefois largement contrôlés par le Conseil, d'autant que celui-ci ne permet pas qu'il soit aussi invoqué par les justiciables dans le cadre des QPC. Rien ne lui interdit qu'il en soit autrement. Le coût d'une ouverture sera sans doute celui de la contradiction avec la décision Société Casuca, qu'un art bien maitrisé de l'argumentation pourrait néanmoins contourner. Car sur le fond, c'est plutôt en cohérence que l'on gagnera: cohérence au regard non seulement de l'ampleur des enjeux qui peuvent être associés à l'objectif de protection de l'environnement (comme celui, analogiquement, de l'objectif d'égal accès des femmes et des hommes aux responsabilités professionnelles), mais aussi des usages contentieux des divers types de normes constitutionnelles.

Conseil constitutionnel, Décision $\mathrm{n}^{\circ}$ 2019-823 QPC du 31 janvier 2020, Union des industries de la protection des plantes. 
Les Lettres « Actualités Droits-Libertés » (ADL) du CREDOF (pour s'y abonner) sont accessibles sur le site de la Revue des Droits de l'Homme (RevDH) - Contact

\section{NOTES}

1. Voir Véronique Champeil-Desplats, «Charte de l'environnement : La QPC bute sur l'incipit », La Revue des droits de l'homme, Actualités Droits-Libertés .

2. Voir Karine Foucher, «L'apport de la QPC au droit de l'environnement », in L'apport de la QPC à la protection des droits et libertés. Un bilan, Dalloz, 2020, Thème et commentaire, pp. 5 et s.

\section{ABSTRACTS}

La décision $\mathrm{n}^{\circ}$ 2019-823 QPC du 31 janvier 2020 fera date. Préambule de la Charte de l'environnement à l'appui, le Conseil constitutionnel y affirme que «la protection de l'environnement, patrimoine commun des êtres humains, constitue un objectif de valeur constitutionnelle ». Voilà qui pourra réjouir toutes celles et tous ceux qui attendent des signes de rééquilibrage entre les libertés économiques, d'un côté, et des exigences à caractère social ou environnemental de l'autre, et ont toujours refusé de réduire la Charte de l'environnement, y compris son préambule, à un texte purement symbolique. Cette consécration de l'utilité juridique de l'incipit augure-t-elle pour autant un changement de conception quant à son invocabilité par des requérants dans le cadre de la QPC? L'invocation du préambule de la charte de l'environnement par le Conseil constitutionnel pour justifier son appréciation de la constitutionnalité de dispositions législatives contestées à l'occasion d'une QPC emporte-t-elle la possibilité de son invocabilité par des justiciables? Rien n'est certain.

\section{AUTHOR}

\section{VÉRONIQUE CHAMPEIL-DESPLATS}

Professeure de droit public, Université Paris Nanterre (CTAD-CREDOF) 